\title{
Intensive patients' care program ameliorates anxiety and depression, and sustained anxiety correlates with worse overall survival in renal cell carcinoma patients underwent radical nephrectomy
}

\author{
Bingbing Song, Yuling Zhang, Yanjie Wang, Xushu An \\ Department of Urology Surgery, Harbin Medical University Cancer Hospital, Harbin 150000, China \\ Contributions: (I) Conception and design: X An; (II) Administrative support: B Song; (III) Provision of study materials or patients: Y Zhang, Y Wang; \\ (IV) Collection and assembly of data: Y Zhang; (V) Data analysis and interpretation: B Song, X An; (VI) Manuscript writing: All authors; (VII) Final \\ approval of manuscript: All authors. \\ Correspondence to: Xushu An. Department of Urology Surgery, Harbin Medical University Cancer Hospital, 150 Haping Road, Harbin 150000 , \\ China. Email: anxushu@126.com.
}

\begin{abstract}
Background: This study aimed to investigate the effect of an intensive patients' care program (IPCP) on anxiety, depression, and survival rate of renal cell carcinoma (RCC) patients post radical nephrectomy.

Methods: This study consisted of two stages: a randomized, controlled stage (stage I) and a longterm follow-up stage (stage II). A total of 182 RCC patients who underwent radical nephrectomy were consecutively recruited and were allotted into a IPCP group (IPCP plus usual care), or a control group (only usual care) in a 1:1 ratio. Anxiety and depression were evaluated by HADS and SAS/SDS at baseline (M0), M3, M6, M9 and M12. Overall survival (OS) was documented.
\end{abstract}

Results: The decrease of HADS-A score was larger, the percentage of patients with anxiety at M12 assessed by HADS-A score was reduced and the percentage of patients with light anxiety at M12 assessed by HADS-A score was greater in IPCP group compared with control group. The SAS score at M3, M6, M9 and M12 were declined and the SAS score reduction at M12 was greater in IPCP group compared with control group. The reduction of HADS-D score in IPCP group was greater compared with control group. The SDS score at M12 was reduced in IPCP group compared with control group, and the decrease of SDS score at M12 in IPCP group was larger compared with control group. Patients with sustained anxiety assessed by HADS-A score and SAS score had worse OS compared with patients without sustained anxiety.

Conclusions: IPCP ameliorated anxiety and depression, and sustained anxiety correlated with worse OS in RCC patients post radical nephrectomy.

Keywords: Intensive care; renal nursing; anxiety; depression

Submitted Jun 04, 2018. Accepted for publication Nov 21, 2018.

doi: $10.21037 /$ tcr.2018.12.07

View this article at: http://dx.doi.org/10.21037/tcr.2018.12.07

\section{Introduction}

Kidney cancer, one of the top ten most frequent cancers worldwide, attacks 668,000 people in China until 2015 according to the epidemiologic data $(1,2)$. Accounting for over $80 \%$ of all kidney cancer cases, renal cell carcinoma (RCC) exhibits a poor prognosis particularly for patients in the advanced stages (3-5). Nephrectomy combined with targeted therapy or adjuvant therapy remains the preferred option for prolonging survival of RCC patients, especially for metastatic RCC patients $(6,7)$. However, with the extension of patients' survival, patients' quality of life is attracting more attention in RCC management. As a critical 


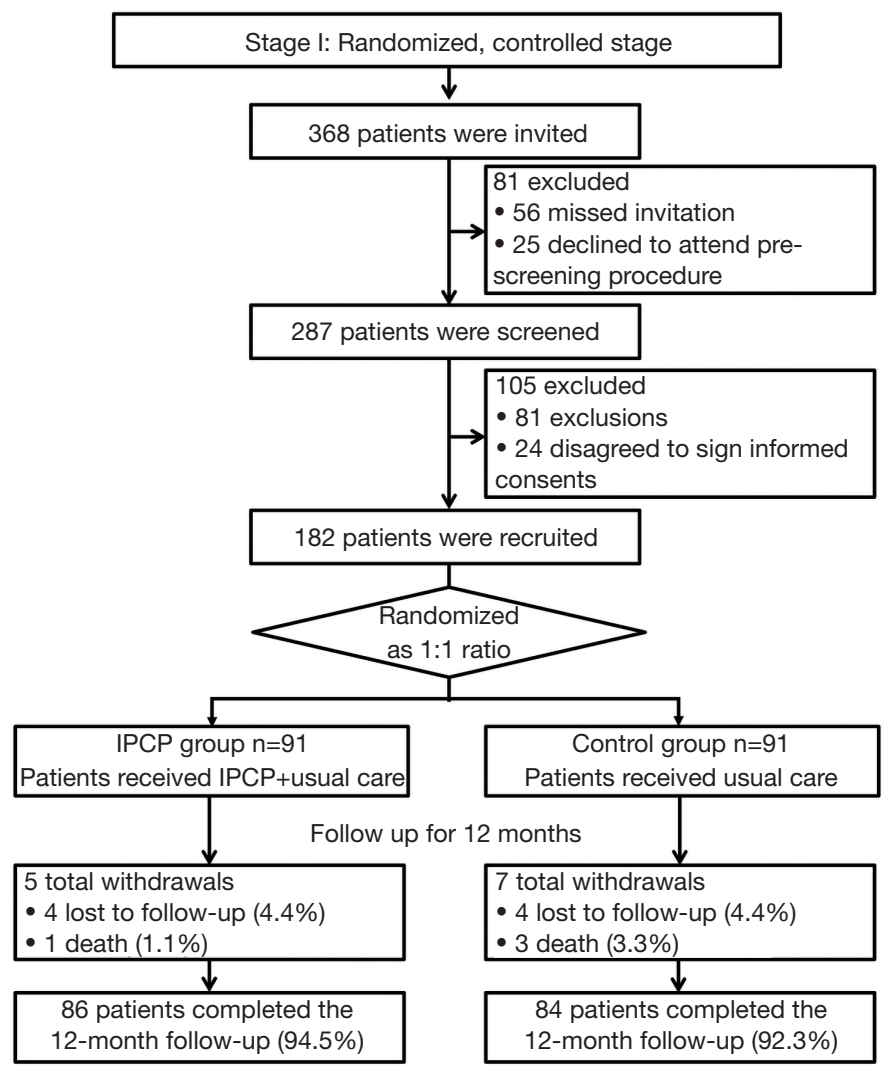

Figure 1 Study flow of stage I.

aspect of quality of life, unstable psychological status has been reported in RCC patients post radical nephrectomy, with depression and anxiety being the most common mood disorders observed in clinical practice $(8,9)$. Although anxiety and depression are not rare in RCC patients, their impact on the outcomes for the patients is still uncertain.

Intensive patients' care program (IPCP) is a novel care program involving several aspects that aims to improve the quality of life of cancer patients, and consists of patients' education, physical training, telephone counseling and emotional support and loving care. Cancer patients form a delicate population with growing quantity that requires special care as there is an increasing number of patients living with cancer, which is a profit from the advancement of the screening program, diagnosis and treatment modalities (10). Intensive care of cancer patients has progressed significantly since it was first adopted in the 1950s and has enhanced the treatment efficacy in cancer patients $(11,12)$. Despite the current benefit received from intensive care, its potential for improving mood disorders and clinical outcomes of RCC patients still remains mostly unexplored.
Thus, the aim of our study was to investigate the effect of IPCP on anxiety, depression and survival of RCC patients post radical nephrectomy.

\section{Methods}

\section{Study design}

The present study consisted of two stages: a randomized, controlled stage (stage I) and a long-term follow-up stage (stage II) (Figures 1,2). In the randomized, controlled stage, eligible patients were randomly allocated into an IPCP group or a control group in a 1:1 ratio. The IPCP group received both IPCP and standard care for 12 months, and the control group received standard care alone for 12 months. In order to assess the effect of IPCP on the improvement of patients' anxiety and depression, the Hospital Anxiety and Depression Scale anxiety/ depression (HADS-A/HADS-D) scores and the Zung SelfRating Anxiety/Depression Scale (SAS/SDS) scores were assessed at baseline (M0), month 3 (M3), M6, M9 and M12. Additionally, intention-to-treat (ITT) analysis was performed based on the last observation carried forward 


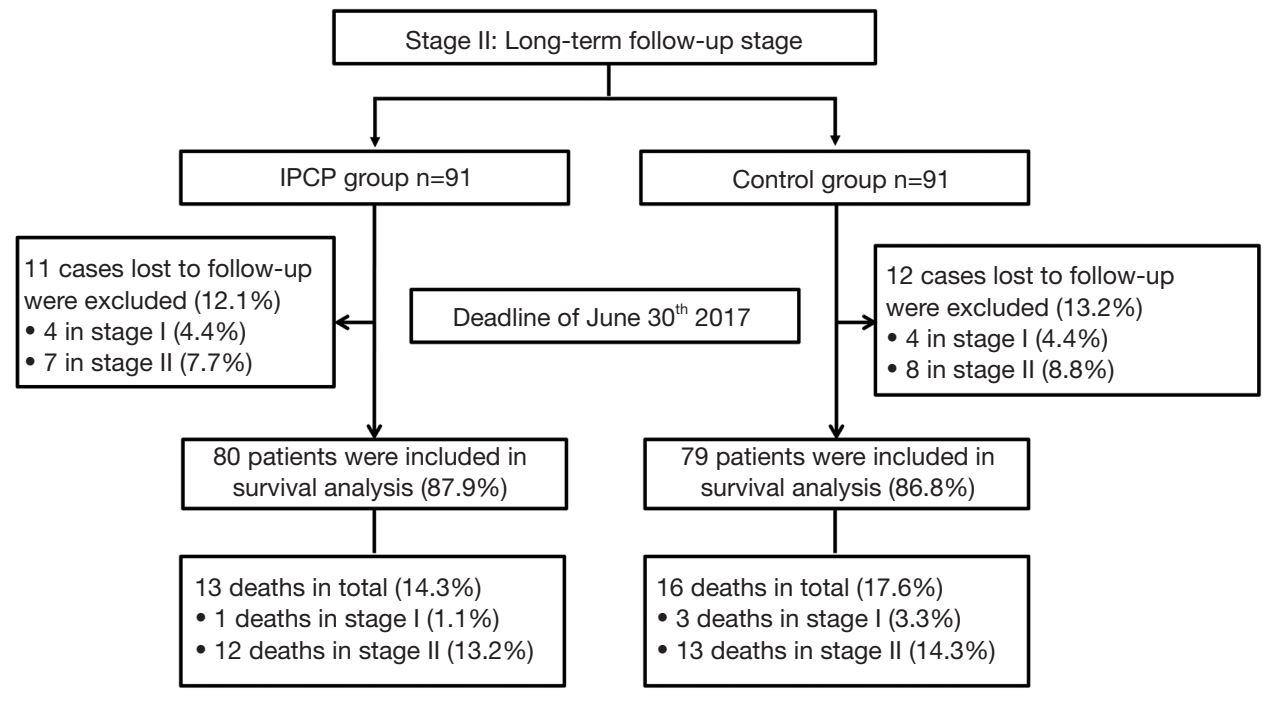

Figure 2 Study flow of stage II.

(LOCF) method. In the long-term follow-up stage, patients were further followed-up with in order to assess their overall survival (OS) rate. The last follow-up date was Jun 30th, 2017 and the median follow-up duration was 43.0 months (IQR: 30.0-54.0 months). Patients who were lost to follow-up were excluded from the final analysis of OS.

\section{Participants}

In this present randomized, controlled study a total of 182 RCC patients who underwent radical nephrectomy at the Harbin Medical University Cancer Hospital between January 2012 and December 2015 were consecutively recruited. The inclusion criteria consisted of the following: (I) diagnosed as RCC according to clinical, imaging and pathological findings; (II) age above 18 years; (III) unilateral renal cell carcinoma; (IV) received radical nephrectomy; (V) able to complete the questionnaire of assessments. Exclusion criteria were the following: (I) received antidepressant or antianxiety treatment within 3 months; (II) complicated with severe mental diseases such as dementia, schizophrenia and so on; (III) uncontrolled hypertension or diabetes; (IV) life expectancy less than 12 months; (V) a history of liver or heart dysfunction or other malignant tumors; (VI) unlikely to be followed up regularly; (VII) pregnant or lactating women. This study was approved by the Ethics Committee of the Harbin Medical University Cancer Hospital with approval number 2011-12$\mathrm{R}$ and conducted according to the Declaration of Helsinki. All participants signed the informed consent.

\section{Data collection}

After enrollment, baseline characteristics were collected from all participants including (I) demographic characteristics: age, gender and highest education; (II) clinical and pathological characteristics: smoking history, alcohol usage, hypertension, hyperlipidemia, diabetes, tumor side, histological subtypes and TNM stage, as evaluated according to the 7 th edition of the American Joint Committee on Cancer (AJCC) cancer staging manual.

\section{Randomization}

Block randomization was used in the present study, and randomization sequence was created using the STATA 9.0 (StataCorp, USA) statistical software with a 1:1 allocation using random block sizes of 6 by a statistical analyzer who did not participate in the recruitment process for assignment. The documents were sent and kept in Shanghai Qeejen Biotech Company (Shanghai, China). After confirmation of each patient's eligibility, a research nurse made a call to the company and a unique subject identification number was given from the randomized module.

\section{Interventions}

The IPCP consisted of four sections which included patients' education, physical training, telephone counseling and emotional support and loving care, which were conducted in the following fashion. (I) Patients' education. In the first week, 
after enrollment, health education materials developed by a related research therapist were dispensed to each patient, which included the postoperative management of RCC, physical training, mental care and so on. Then, sessions of education and detailed instructions were given to the patients by nurses once a week in the first month. (II) Physical training. The physical training included two parts: low-intensity physical training and moderate-intensity physical training. At the beginning of each part, patients received the specific training and instructions once a week for one month given by trained research nurses at the Rehabilitation Center. Then, a moderateintensity home-based exercise plan was made and achievable goals were set with the help of professional nurses, and carried out at home. Both parts were performed for 6 months. The physical training details are shown in Supplementary Table S1. (III) Telephone counseling. After the education in the first month, each patient was assigned a health counselor (a study-trained health nurse), and counseling sessions were conducted every 2 weeks for 12 months. The duration of each telephone session was 25-30 minutes. During each telephone call, the counselor monitored self-reported progress, explored strategies for overcoming barriers, fielded questions and encouraged patients to persist in physical training. (IV) Emotional support and loving care. As the prior caregivers, patients and family members were invited to join in the monthly workshop, during which nurses would engage with them in genuine communication, and try their best to resolve patients' troubles and issues as well as give detailed advice to the patients about how to consume a healthy diet, keep a good mood etc.

The standard care included instructions for the postoperative medical management of RCC, regular examinations [computed tomography (CT), magnetic resonance imaging (MRI) or ultrasonography (US) every 3 months] and the usual recommendations for postoperative rehabilitation.

\section{Endpoints}

The primary endpoint was the change of HADS-A, SAS, HADS-D and SDS scores at M12 compared to baseline. The secondary endpoints included the following: (I) the percentage of patients with anxiety determined by HADS-A score or SAS score, and the percentage of patients with depression assessed by HADS-D score or SDS score at M12; (II) the OS of each group.

\section{Definitions}

The HADS-A/HADS-D and SAS/SDS scores were applied to evaluate the anxiety and depression of patients. Both HADS-A and HADS-D consisted of 7 questions which were scored as $0-3$ points individually, totaling $0-21$ points with the following score classifications: $0-7$, no anxiety/ depression; 8-10, light anxiety/depression; 11-14, moderate anxiety/depression; 15-21, severe anxiety/depression (1). Similarly, both the SAS and SDS assessments contained 20 questions which were scored as 1-4 points individually, ranging from "none or a little of the time" to "most or all of the time", resulting in a 20-80 raw score. Subsequently, standard scores were calculated by int $\left(1.25^{*}\right.$ raw score $)$ and assigned to the following classifications: $25-49$, no anxiety/ depression; 50-59, light anxiety/depression; 60-69, moderate anxiety/depression; 70-100, severe anxiety/depression (2,3). Sustained anxiety patients were defined as patients with anxiety at M0 and M12 assessed by the HADS-A or SAS score. Similarly, sustained depression patients were defined as patients with depression at M0 and M12 assessed by the HADS-D or SDS score. In addition, OS was defined as the time of intervention to the date of death from any cause.

\section{Statistics}

Statistical analysis was performed using the Stata 9.0 (StataCorp, USA) software, SPSS 22.0 software (IBM, USA) and Graphpad Prism 6 software (GraphPad Software Inc, USA). Data were mainly presented as a mean value \pm the standard deviation or count (percentage). Comparison between the two groups was determined by $t$-test, Chisquare test or Wilcoxon rank sum test. Kaplan-Meier (K$\mathrm{M})$ curve and log-rank test were used to analyze the OS between the two groups. $\mathrm{P}<0.05$ was considered significant.

\section{Results}

\section{Study flow}

This study included two stages: the randomized, controlled stage (stage I) and the long-term follow-up stage (stage II).

At stage I, 368 RCC patients were initially invited, although 81 patients were excluded due to missing invitations ( $\mathrm{N}=56$ ) and declined to attend pre-screening procedure; thus, the remaining 287 patients received screening for eligibility. Additionally, 105 patients, including 81 exclusions and 24 patients, were excluded because they did not agree to sign informed consent forms. Finally, there were 182 patients who were recruited in our study and subsequently allocated in a 1:1 ratio into the IPCP group $(\mathrm{N}=91)$ and the control group (N=91). In the IPCP group, patients received IPCP combined with standard care, and 5 patients were withdrawn from the study due to 4 being lost to follow-up (4.4\%) and 1 from death $(1.1 \%)$. The remaining 86 patients completed the 
Table 1 Revised baseline characteristics of patients in IPCP group and control group

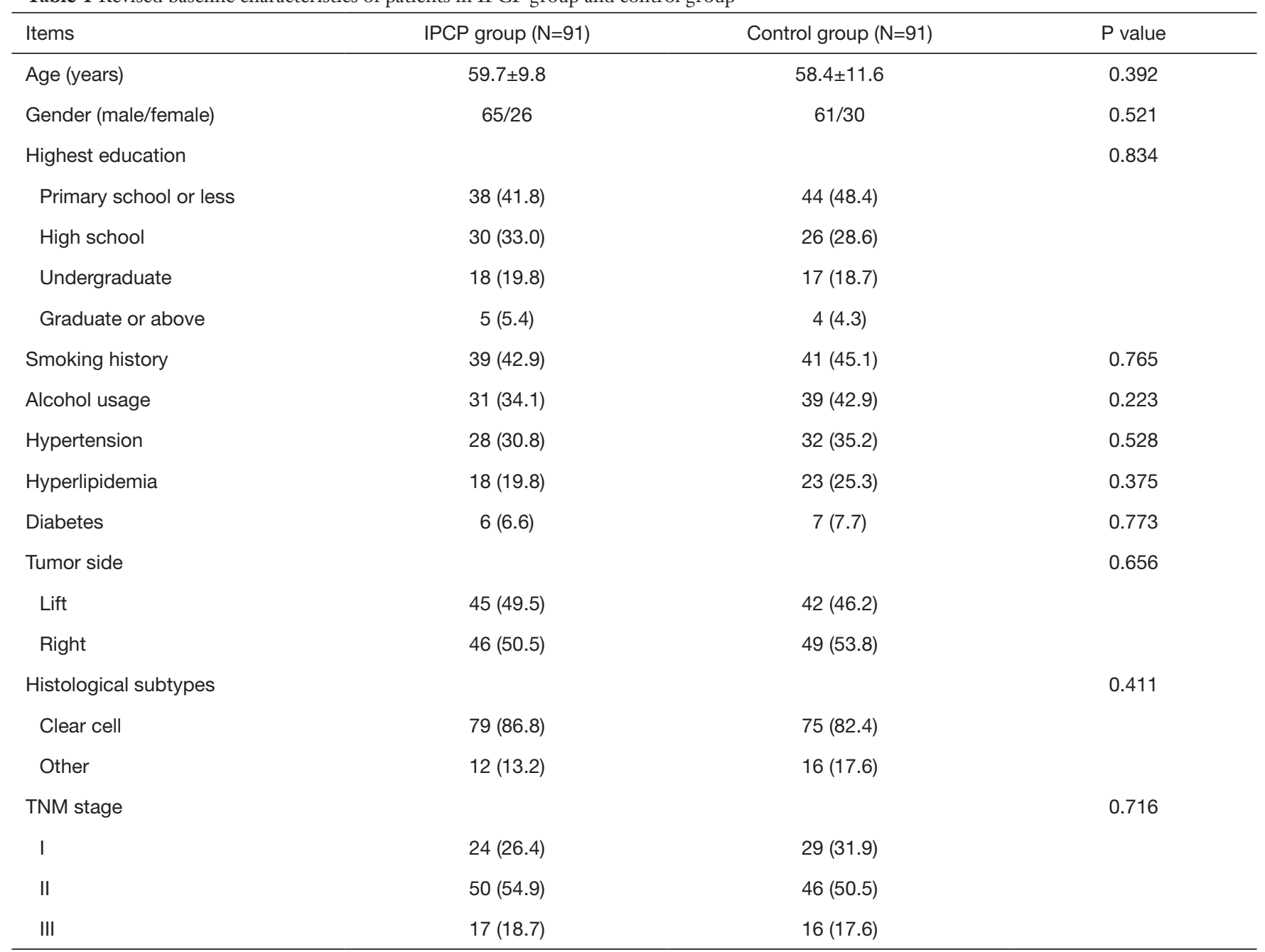

Data were presented as mean value \pm standard deviation or count (percentage). Comparison was determined by $t$-test or Chi-square test.

$P$ value $<0.05$ was considered significant. IPCP, intensive patient's care program.

12-month follow-up (94.5\%). In the control group, in which patients received only standard care, there were 7 withdrawals consisting of 4 patients who were lost to follow-up (4.4\%) and 3 deaths (3.3\%).

At stage II, 11 cases who were lost during the follow-up period were excluded (12.1\%) from the IPCP group [ 4 cases in stage I (4.4\%) and 7 cases in stage II (7.7\%)], and 12 cases who were lost to follow-up were excluded (13.2\%) from the control group [ 4 cases in stage I (4.4\%) and 8 cases in stage II $(8.8 \%)]$. Subsequently, in the IPCP group, 80 patients were included in the survival analysis $(87.9 \%)$, among whom 13 patients died (14.3\%) including 1 death in stage I (1.1\%) and 12 deaths in stage II (13.2\%). As for the control group, there were 79 patients included in the survival analysis $(86.8 \%)$ and the number of deaths was 16 , consisting of 3 deaths in stage I
(3.3\%) and 13 deaths in stage II (14.3\%).

\section{Baseline characteristics of patients in IPCP group and control group}

As presented in Table 1, the mean age of patients was $59.7 \pm 9.8$ years in the IPCP group and was $58.4 \pm 11.6$ years in the control group $(\mathrm{P}=0.392)$. There were 65 males and 26 females in the IPCP group, and 61 males and 30 females in the control group $(\mathrm{P}=0.521)$. The numbers of patients with the highest level of education being primary school or less, high school, undergraduate and graduate or above were $38(41.8 \%), 30(33.0 \%), 18(19.8 \%)$ and $5(5.4 \%)$ in the IPCP group, and were 44 (48.4\%), 26 (28.6\%), 17 (18.7\%) and $4(4.4 \%)$ in the control group $(\mathrm{P}=0.834)$. Thirty-nine 
(42.9\%) patients in the IPCP group and 41 (45.1\%) patients in the control group had a smoking history $(\mathrm{P}=0.765)$. The number of patients who had used alcohol in the IPCP group and control group were $31(34.1 \%)$ and 39 (42.9\%), respectively ( $\mathrm{P}=0.223)$. In the IPCP group, 24 (26.4\%) patients were in TNM stage I, 50 (54.9\%) patients were in TNM stage II and 17 (18.7\%) patients were in TNM stage III, and the numbers of patients in TNM stage I, II and III in the control group were 29 (31.9\%), 46 (50.5\%) and 16 $(17.6 \%)$, respectively $(\mathrm{P}=0.716)$. The information on other clinicopathological characteristics was listed in Table 1.

\section{Baseline assessments of anxiety and depression of patients in the IPCP group and control group}

At the baseline, the HADS-A score $(\mathrm{P}=0.711)$, percentage of patients with anxiety by HADS-A score $(\mathrm{P}=0.746)$, anxiety severity by HADS-A score $(\mathrm{P}=0.957)$, SAS score $(\mathrm{P}=0.401)$, percentage of patients with anxiety assessed by SAS score $(\mathrm{P}=0.620)$, anxiety severity by SAS score $(\mathrm{P}=0.969)$, HADS-D score $(\mathrm{P}=0.451)$, proportion of patients with depression assessed by HADS-D score $(\mathrm{P}=0.864)$, depression severity by HADS-D score $(\mathrm{P}=0.158)$, SDS score $(\mathrm{P}=0.579)$, proportion of patients with depression assessed by SDS score $(\mathrm{P}=0.866)$ and depression severity by SDS score $(\mathrm{P}=0.962)$ in the IPCP group showed no difference compared with those in the control group (Table 2).

\section{The change of anxiety of patients in the IPCP group and control group}

As shown in Figure 3, there was no difference of HADS-A score at M0, M3, M6, M9 or M12 between the IPCP group and control group (all $\mathrm{P}>0.05$ ) (Figure $3 A$ ). However, the decrease of HADS-A score $(\mathrm{P}=0.019$, Figure $3 B)$ was larger and the percentage of patients with anxiety assessed by HADS-A score at M12 ( $\mathrm{P}=0.032$, Figure 3C) was reduced in the IPCP group compared with the control group. In addition, at M12, the percentage of patients with light anxiety was higher, the proportion of patients with moderate anxiety was lower, and the percentage of patients with severe anxiety was higher in the IPCP group compared with the control group $(\mathrm{P}=0.027$, Figure $3 D)$.

As for anxiety assessed by the SAS score, there was no difference in the SAS scores in the IPCP group compared with the control group at $\mathrm{M} 0(\mathrm{P}>0.05)$; however, the SAS scores at M3 $(\mathrm{P}<0.05), \mathrm{M} 6(\mathrm{P}<0.01), \mathrm{M} 9(\mathrm{P}<0.001)$ and $\mathrm{M} 12$ $(\mathrm{P}<0.001)$ in the IPCP group were lower compared with the control group (Figure 4A), while the SAS scores in the IPCP group showed greater reduction than in the control group $(\mathrm{P}=0.019$, Figure $4 B)$. Meanwhile, the percentage of patients with anxiety assessed by SAS score at M12 $(\mathrm{P}=0.091$, Figure $4 C$ ) and percentage of patients with different anxiety severity assessed by SAS score at M12 $(\mathrm{P}=0.084$, Figure $4 D)$ were similar between the IPCP group and the control group.

\section{The change of depression of patients in IPCP group and control group}

The reduction of HADS-D score in the IPCP group was greater than in the control group $(\mathrm{P}=0.032$, Figure $5 A)$. However, the HADS-D scores at M0, M3, M6, M9 and M12 (all $\mathrm{P}>0.05$, Figure $5 B$ ), the percentage of patients with depression assessed by HADS-D score at M12 ( $\mathrm{P}=0.342$, Figure 5 C) and the percentage of patients with different depression severity assessed by HADS-D score at M12 $(\mathrm{P}=0.335$, Figure $5 D)$, were similar between the two groups.

As displayed in Figure 6A, the SDS score at M12 was reduced in IPCP group compared with the control group $(\mathrm{P}<0.05)$, while there was no difference of the SDS scores at M0, M3, M6 or M9 found between the two groups (all $\mathrm{P}>0.05$ ). Additionally, the decrease of the SDS score at M12 in the IPCP group was larger compared with the control group $(\mathrm{P}=0.018$, Figure $6 B)$. No difference in the percentage of patients with depression assessed by SDS score $(\mathrm{P}=0.188$, Figure $6 C$ ) or percentage of patients with different depression severity assessed by SDS score at M12 (P=0.257, Figure $6 D$ ) was discovered between the two groups.

\section{Survival of patients in IPCP group and control group}

As displayed in Figure 7, the OS of patients in the IPCP group was similar to that of the control group $(\mathrm{P}=0.218)$, and the mean OS of the IPCP group and control group were 57.5 months (95\% CI: 54.6-60.3 months) and 55.1 months (95\% CI: 51.2-59.0 months), respectively.

\section{The influence of sustained anxiety or depression on survival}

Patients with sustained anxiety assessed by the HADS-A score had worse OS compared with that of patients without sustained anxiety assessed by HADS-A score $(\mathrm{P}=0.026$, Figure $8 \mathrm{~A}$ ), and patients with sustained anxiety assessed by SDS score also had a shorter OS compared with patients without sustained anxiety assessed by SAS score $(\mathrm{P}=0.012$, Figure $8 B)$. However, no difference of OS between patients with or without depression assessed by HADS-D score $(\mathrm{P}=0.166$, Figure $8 C)$ or SDS score $(\mathrm{P}=0.131$, Figure $8 D)$ was found. In addition, the baseline demographic and clinicopathological characteristics between patients with 
Table 2 Baseline assessments of anxiety and depression of patients in IPCP group and control group

\begin{tabular}{|c|c|c|c|}
\hline Items & IPCP group $(\mathrm{N}=91)$ & Control group (N=91) & $P$ value \\
\hline No anxiety (HADS-A score 0-7) & $65(71.4)$ & $63(69.2)$ & 0.746 \\
\hline Anxiety (HADS-A score 8-21) & $26(28.6)$ & $28(30.8)$ & \\
\hline Anxiety severity by HADS-A score & & & 0.957 \\
\hline Moderate (HADS-A score 11-14) & $6(6.6)$ & $8(8.8)$ & \\
\hline Severe (HADS-A score 15-21) & $2(2.2)$ & $2(2.2)$ & \\
\hline SAS score & $44.3 \pm 10.2$ & $45.5 \pm 9.9$ & 0.401 \\
\hline No anxiety (SAS score 25-49) & $67(73.6)$ & $64(70.3)$ & 0.620 \\
\hline Light (SAS score 50-59) & $15(16.5)$ & $17(18.7)$ & \\
\hline Moderate (SAS score 60-69) & $8(8.8)$ & $9(9.9)$ & \\
\hline Severe (SAS score 70-100) & $1(1.1)$ & $1(1.1)$ & \\
\hline HADS-D score & $6.3 \pm 2.8$ & $5.9 \pm 3.6$ & 0.451 \\
\hline No depression (HADS-D score $0-7$ ) & $68(74.7)$ & $69(75.7)$ & 0.864 \\
\hline Depression (HADS-D score 8-21) & $23(25.3)$ & $22(24.3)$ & \\
\hline Depression severity by HADS-D score & & & 0.158 \\
\hline Light (HADS-D score 8-10) & $18(19.8)$ & $10(11.1)$ & \\
\hline Depression severity by SDS score & & & 0.962 \\
\hline Light (SDS score 50-59) & $14(15.4)$ & $15(16.5)$ & \\
\hline Moderate (SDS score 60-69) & $9(9.9)$ & $7(7.7)$ & \\
\hline Severe (SDS score 70-100) & $1(1.1)$ & $1(1.1)$ & \\
\hline
\end{tabular}

Data were presented as mean value \pm standard deviation or count (percentage). Comparison was determined by $t$-test or Chi-square test. $P$ value $<0.05$ was considered significant. IPCP, intensive patient's care program; HADS-A score, Hospital Anxiety and Depression Scale anxiety score; HADS-D score, Hospital Anxiety and Depression Scale depression score; SAS score, Zung Self-Rating Anxiety Scale; SDS score, Zung Self-Rating Depression Scale.

and without sustained anxiety were compared, which revealed that there was no difference in all the baseline characteristics between patients with sustained anxiety and patients without sustained anxiety assessed by HADS-A score (Table S2) or SAS score (Table S3) (all P>0.05).

\section{Discussion}

In our study, the results elucidated that: (I) the anxiety condition assessed by HADS-A score and SAS score was ameliorated in the IPCP group compared with the control 

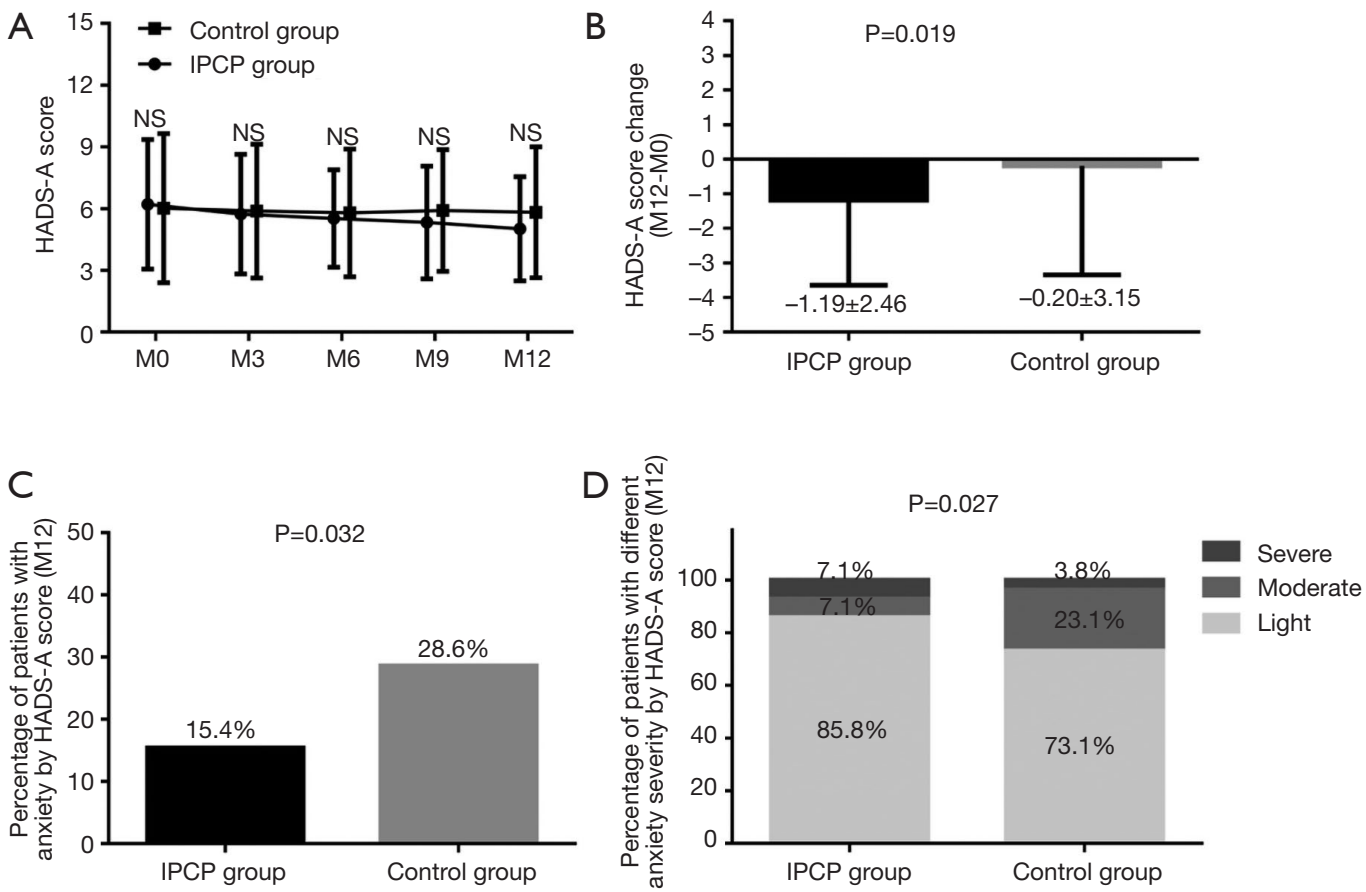

Figure 3 Change of anxiety assessed by HADS-A score. No difference of HADS-A score at M0, M3, M6, M9 or M12 in IPCP group and control group was found (A), while the reduction of HADS-A score (B) was larger and the percentage of patients with anxiety assessed by HADS-A score at M12 (C) was reduced in the IPCP group compared with the control group. At M12, the percentage of patients with light anxiety was more massive, the proportion of patients with moderate anxiety was decreased and the percentage of patients with severe anxiety was increased in the IPCP group compared with the control group (D). Comparison between the two groups was determined by $t$-test, Wilcoxon rank sum test or Chi-square test. $\mathrm{P}<0.05$ was considered significant. HADS, Hospital Anxiety and Depression Scale; IPCP, intensive patients' care program; NS, not significant.

group, while the depression condition assessed by HADS-D score and SDS score was also alleviated in the IPCP group compared with the control group; (II) the OS in the IPCP group was similar to that in the control group, although patients with sustained anxiety assessed by HADS-A score and SAS score had a worse OS compared with those patients with no anxiety assessed by HADS-A score or SAS score.

Patients with cancer account for a vulnerable population that needs intensive care to improve or at least retains their quality of life, and the growing number of cancer survivors has led to a substantial unmet need in the field of supportive oncology care. Accumulating cases of anxiety and depression in cancer patients have been reported by previous studies, and the roles of anxiety and depression in patients with kidney cancers, including RCC, have become a crucial subject of clinical researches over the past few years $(8,13)$. The influence of intensive care on ameliorating anxiety and depression in RCC patients is under-investigated, however, the findings in other cancers have provided some insight into this area. A previous longitudinal study conducted on cancer patients who accept psychosocial care illustrates that patients present with elevated goal disengagement post psychosocial care, and a higher goal reengagement correlates with fewer symptoms of anxiety and depression (14). And a randomized controlled trial elucidates that a tailored telephone intervention reduces the incidence of increased anxiety in colorectal cancer patients (15). These results are partially in accordance with ours, which illustrated that the anxiety and depression assessed by HADS scale and SAS/ SDS scale of RCC patients who received radical nephrectomy were reduced in patients receiving IPCP compared with patients receiving only standard care, indicating that IPCP did mitigate anxiety and depression in RCC patients after radical nephrectomy.

These results can be explained with reference to the four predominant contents of IPCP: (I) Patients' education: for one, the patients' education provided professional information on the postoperative care of RCC patients, which ensured the quality of care given to patients. Additionally, the patients' education assured the success of IPCP by giving guidance and instruction for the 

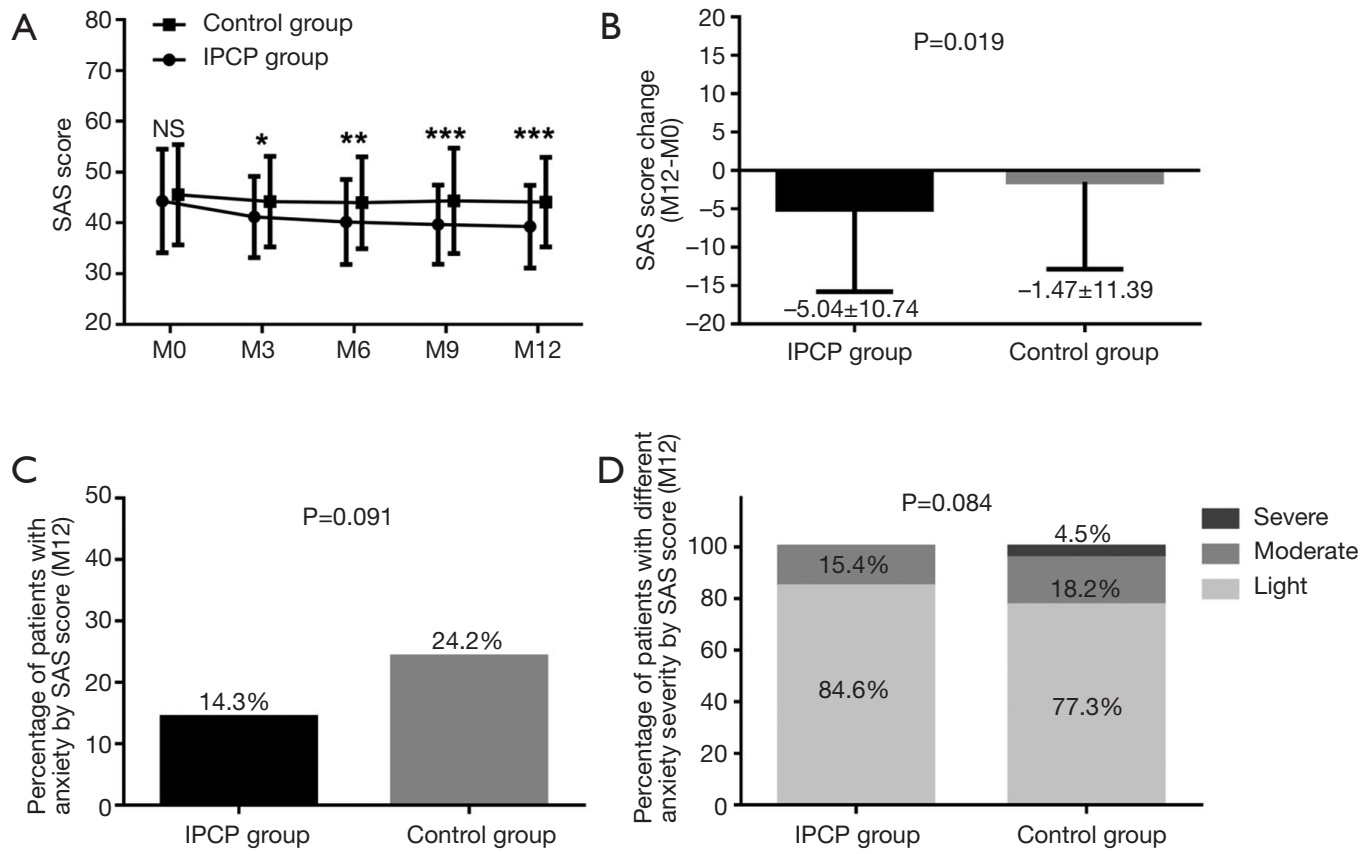

Figure 4 Change of anxiety assessed by SAS score. No difference was discovered in the SAS score of the IPCP group compared with the control group at M0, but the SAS scores at M3, M6, M9 and M12 in the IPCP group were reduced compared with the control group (A). In addition, the decrease of the SAS score in the IPCP group was greater than that in the control group (B). The percentage of patients with anxiety at the M12 assessed by SAS score (C) and percentage of patients with different anxiety severity assessed by SAS score (D) were similar between the IPCP group and control group. Comparison between the two groups was determined by $t$-test, Wilcoxon rank sum test or Chi-square test. $\mathrm{P}<0.05$ was considered significant. *, $\mathrm{P}<0.05$; **, $\mathrm{P}<0.01$; **, $\mathrm{P}<0.001$. SAS, Zung Self-Rating Anxiety Scale; IPCP, intensive patients' care program.

remaining three domains of IPCP to patients and their family members. (II) Physical training: growing evidence elucidates that physical activity improves clinical outcome of cancer patients, more importantly, there is also proof notes that the anxiety and depression are reduced in cancer patients doing physical exercise post treatments (16-18). Although it is still not well understood why physical activity would alleviate the depression and anxiety level in cancer patients, there are several theories which might shed some light on this question. Studies illuminate that physical activity diminishes the distress of cancer patients through raising self-esteem and quality of life, which could consequently lessen the mood disorders of patients (19). In addition, our physical training contains only low and moderate intensity which has been observed to be beneficial to mood disorder improvement through regulating the endocrine system; meanwhile, high level physical activity that has been demonstrated to increase the risk of upper respiratory infection was not included. The low and moderate intensity physical training could be beneficial to mood disorder improvement through regulating the endocrine system (20-22); (III) telephone counseling: telephone counseling was given to each patient by welltrained nurses every 2 weeks for 12 months with the aim of monitoring the progress of the IPCP program. The counselling served the additional purpose of providing solutions from a professional perspective to problems that patients faced during processing the program, which also ensured the consistency of the program. (IV) Emotional support and loving care: first, based on ample evidence, the support of psychosocial oncologists is recommended to be provided to cancer survivors by the American Cancer Society Colorectal Cancer Survivorship Care Guidelines, which illustrates that cancer survivors should be receiving professional care to improve their psychosocial wellbeing $(23,24)$. Second, giving sufficient information on cancer care and the emotional support of cancer caregivers has a positive influence on alleviating the mood disorders of the caregivers, which could subsequently improve the anxiety and depression of RCC patients at the same time due to their communication with each other (25).

Another result in our study illuminates that although the 

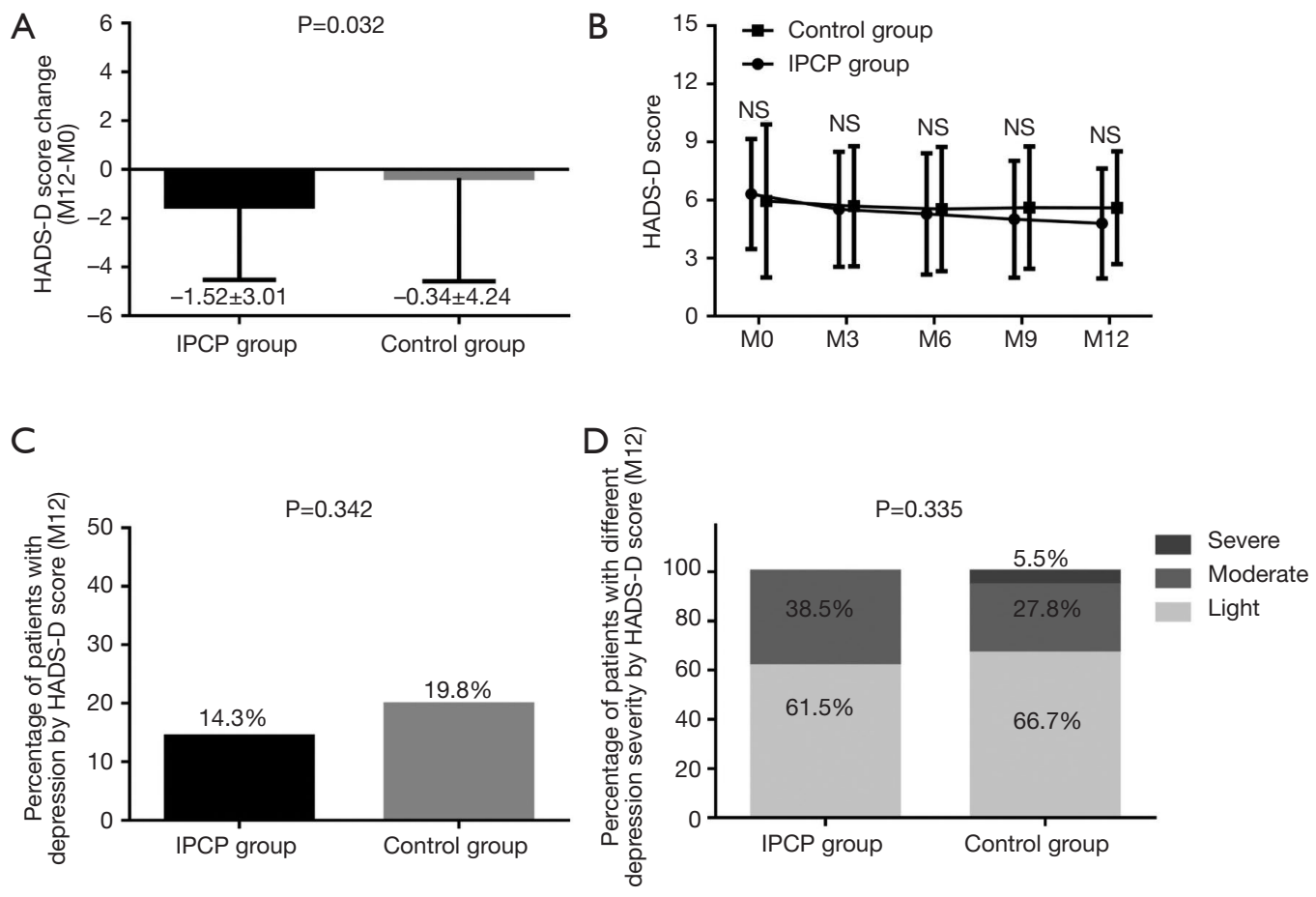

Figure 5 Change of depression assessed by HADS-D score. The decrease of HADS-D score was larger (A), however, the HADS-D score at M0, M3, M6, M9 and M12 (B), the percentage of patients with depression assessed by HADS-D score at M12 (C) and the percentage of patients with different depression severity assessed by HADS-D score (D), showed no differences between the IPCP group compared with the control group. Comparison between the two groups was determined by $t$-test, Wilcoxon rank sum test or Chi-square test. $\mathrm{P}<0.05$ was considered significant. HADS, Hospital Anxiety and Depression Scale; IPCP, intensive patients' care program; NS, not significant.

OS of patients in the IPCP group and control group was of no difference, the OS was worse in patients with sustained anxiety assessed by HADS-A score and SAS score. To the best of our knowledge, the influence of anxiety on survival of RCC patients has not been investigated until now; however, there are indeed several studies that have been conducted in other cancers which have emphasized this issue. An observational study elucidates that the anxiety level assessed by Hamilton Anxiety Rating Scale and serum catecholamines levels positively correlate with worse OS of hepatocellular carcinoma patients after tumor resection (26). Another cohort study based on a large population sample also demonstrates a predictive value of anxiety for high mortality rate in patients with stage III non-small cell lung cancer (27). Partly in accordance with the previous studies, we found that RCC patients had worse OS with sustained anxiety, and there are some possible explanations for these results in our study. Experiments conducted on animal models exhibit an effect of psychological disorders, induced by adding stress on animal models, on the progression of tumors. For instance, it is found that the immobilization stress on rat models accelerates the prevalence and growth of tumor (28). And some other studies also note that stress induced by multiple methods promotes the progression of carcinogenesis through regulating pathways related to hormones, circadian dysregulation and viral oncogenesis (29-33). Although a difference in OS between patients receiving IPCP and standard care was not found in our study, patients with sustained anxiety were observed to have worse OS compared with patients who had no sustained anxiety. In the view that IPCP notably alleviated the anxiety of patients in our study, it is reasonable for us to believe that IPCP improved the survival through ameliorating anxiety in RCC patients post nephrectomy.

Several limitations of this study should be noted. First, in the randomized, controlled stage, the blind method was not used to avoid observer bias. Second, the sample size of 182 RCC patients was relatively small. Third, the type of nephrectomy might have an influence on the psychological status of RCC patients, which should be evaluated.

\section{Conclusions}

In conclusion, IPCP ameliorated anxiety and depression, 

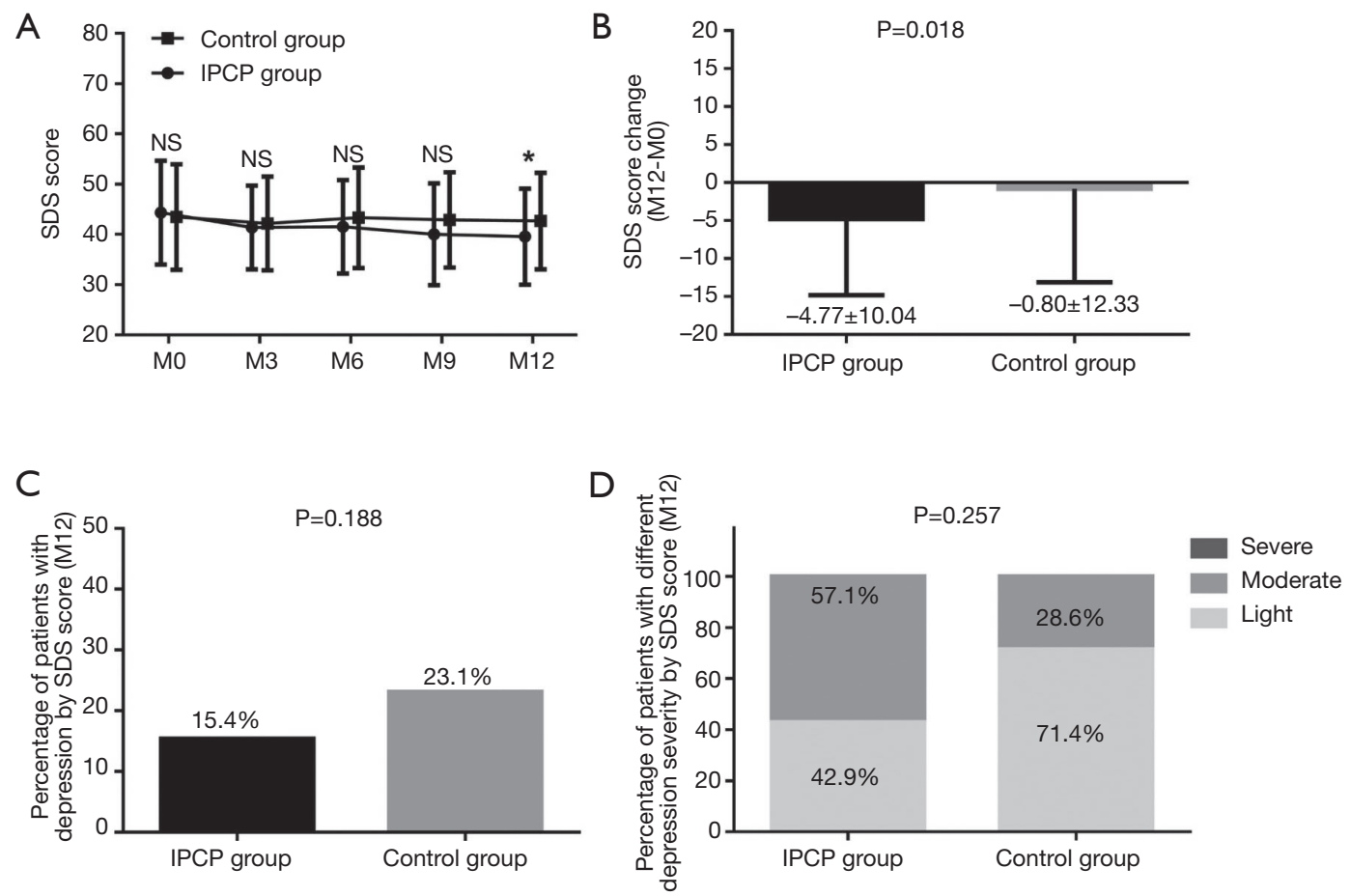

Figure 6 Change of depression assessed by SDS score. The SDS score at M12 was reduced in the IPCP group compared with the control group (A), and the decrease of the SDS score at M12 in IPCP group was larger compared with the control group (B). No difference was found in the percentage of patients with depression assessed by SDS score (C) or percentage of patients with different depression severity assessed by SDS score (D) between the two groups. Comparison between two groups was determined by $t$-test, Wilcoxon rank sum test or Chi-square test. $\mathrm{P}<0.05$ was considered significant. *, $\mathrm{P}<0.05$. SDS, Zung Self-Rating Depression Scale; IPCP, intensive patients' care program.

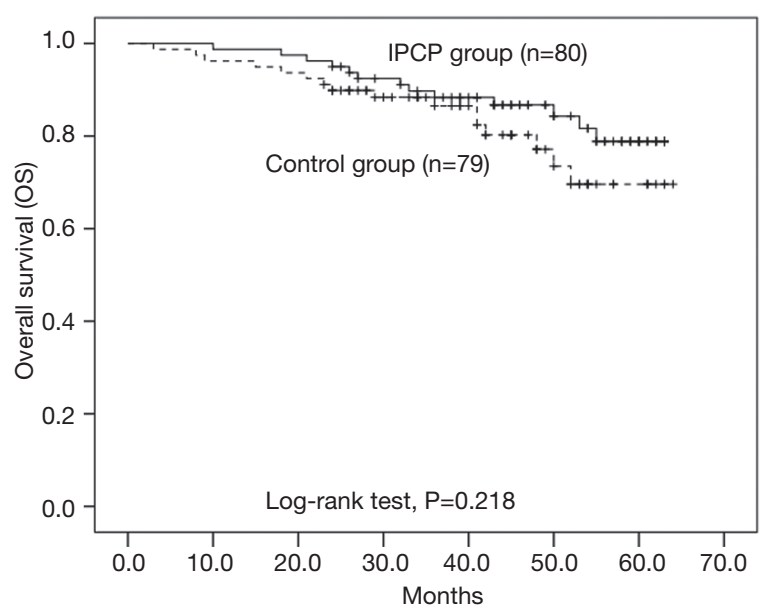

Figure 7 OS in the IPCP group and the control group. The OS in the IPCP group was of no difference in the IPCP group compared with the control group. K-M curve and log-rank test were performed to evaluate the OS of patients in the IPCP group and the control group. $\mathrm{P}<0.05$ was considered significant. OS, overall survival; IPCP, intensive patients' care program; K-M, KaplanMeier. and sustained anxiety correlated with worse OS in RCC patients post radical nephrectomy.

\section{Acknowledgments}

Funding: None.

\section{Footnote}

Conflicts of Interest: All authors have completed the ICMJE uniform disclosure form (available at http://dx.doi. org/10.21037/tcr.2018.12.07). The authors have no conflicts of interest to declare.

Ethical Statement: The authors are accountable for all aspects of the work in ensuring that questions related to the accuracy or integrity of any part of the work are appropriately investigated and resolved. This study was approved by the Ethics Committee of the Harbin Medical University Cancer Hospital with approval number 2011-12$\mathrm{R}$ and conducted according to the Declaration of Helsinki. 

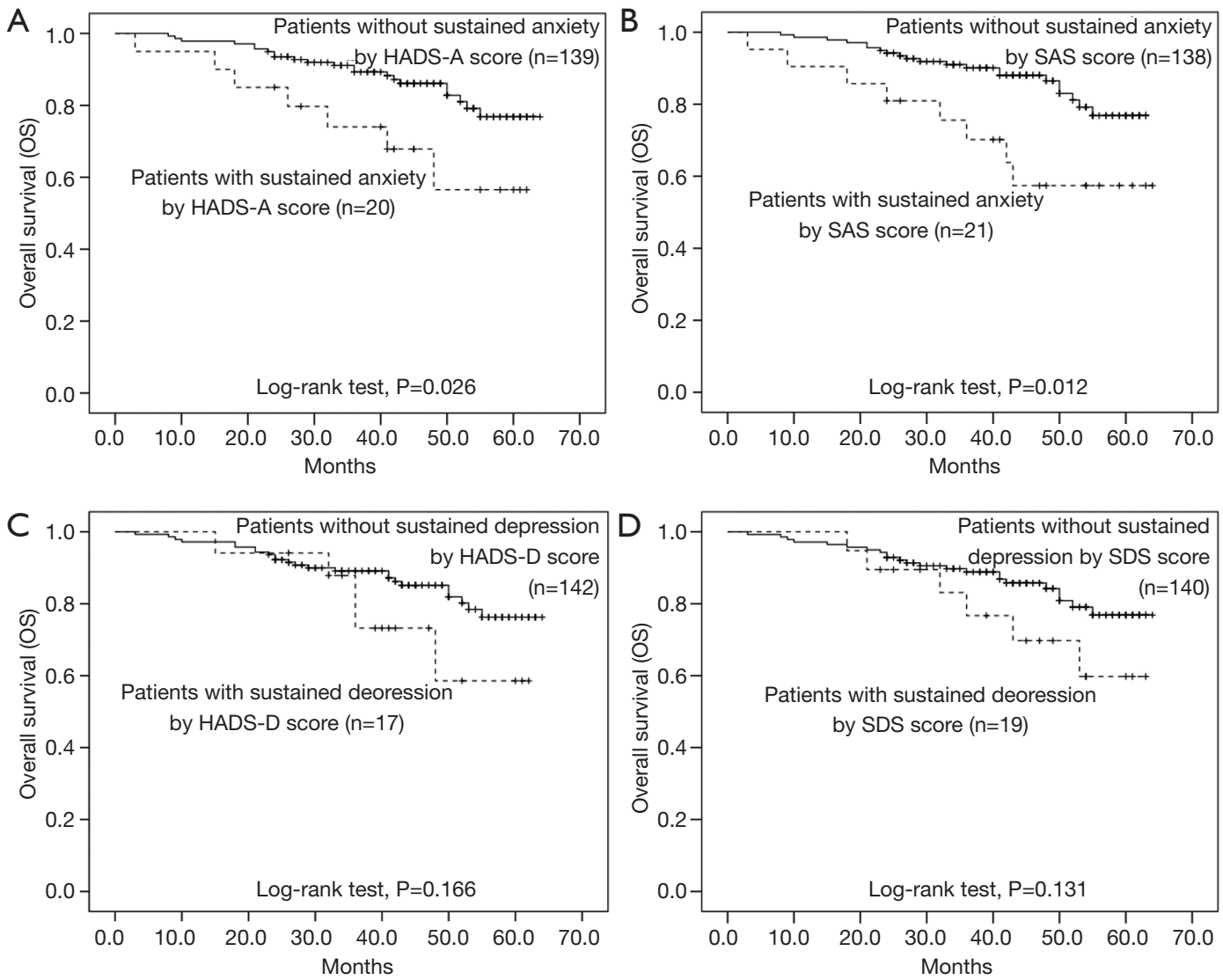

Figure 8 OS of patients with or without sustained anxiety/depression. The OS of patients with sustained anxiety assessed by HADS-A score was worse compared with that of patients without sustained anxiety assessed by HADS-A score (A), and patients with sustained anxiety assessed by SAS score had shorter OS compared with patients without sustained anxiety assessed by SAS score as well (B). However, the OSs between patients with or without depression assessed by HADS-D score (C) or SDS score (D) were similar. K-M curve and log-rank test were performed to evaluate the OS of patients in the IPCP group and control group. $\mathrm{P}<0.05$ was considered significant. OS, overall survival; HADS, Hospital Anxiety and Depression Scale; SAS, Zung Self-Rating Anxiety Scale; SDS, Zung Self-Rating Depression Scale; IPCP, intensive patients' care program; K-M, Kaplan-Meier.

(as revised in 2013) All participants signed the informed consent.

Open Access Statement: This is an Open Access article distributed in accordance with the Creative Commons Attribution-NonCommercial-NoDerivs 4.0 International License (CC BY-NC-ND 4.0), which permits the noncommercial replication and distribution of the article with the strict proviso that no changes or edits are made and the original work is properly cited (including links to both the formal publication through the relevant DOI and the license). See: https://creativecommons.org/licenses/by-nc-nd/4.0/.

\section{References}

1. Chen $W$, Zheng R, Baade PD, et al. Cancer statistics in
China, 2015. CA Cancer J Clin 2016;66:115-32.

2. Torre LA, Bray F, Siegel RL, et al. Global cancer statistics, 2012. CA Cancer J Clin 2015;65:87-108.

3. Siegel RL, Miller KD, Jemal A. Cancer Statistics, 2017. CA Cancer J Clin 2017;67:7-30.

4. Siegel RL, Miller KD, Jemal A. Cancer statistics, 2016. CA Cancer J Clin 2016;66:7-30.

5. Howlader N, Noone AM, Krapcho M, et al. SEER Cancer Statistics Review, 1975-2013, National Cancer Institute. Bethesda, MD, https://seer.cancer.gov/csr/1975_2013/, based on November 2015 SEER data submission, posted to the SEER web site, April 2016.

6. Hong X, Li F, Tang K, et al. Prognostic value of cytoreductive nephrectomy combined with targeted therapy for metastatic renal cell carcinoma: a metaanalysis. Int Urol Nephrol 2016;48:967-75. 
7. Shahait M, Mukherji D, El-Hout Y. Partial nephrectomy for metastatic renal cell carcinoma: Where do we stand? Indian J Urol 2015;31:102-5.

8. Ficarra V, Novella G, Sarti A, et al. Psycho-social well-being and general health status after surgical treatment for localized renal cell carcinoma. Int Urol Nephrol 2002;34:441-6.

9. Van Gool AR, Verkerk R, Fekkes D, et al. Plasma activity of prolyl endopeptidase in relation to psychopathology during immunotherapy with IFN-alpha in patients with renal cell carcinoma. J Interferon Cytokine Res 2008;28:283-6.

10. Masters GA, Krilov L, Bailey HH, et al. Clinical cancer advances 2015: Annual report on progress against cancer from the American Society of Clinical Oncology. J Clin Oncol 2015;33:786-809.

11. Peigne V, Rusinova K, Karlin L, et al. Continued survival gains in recent years among critically ill myeloma patients. Intensive Care Med 2009;35:512-8.

12. Algrin C, Faguer S, Lemiale V, et al. Outcomes after intensive care unit admission of patients with newly diagnosed lymphoma. Leuk Lymphoma 2015;56:1240-5.

13. Yang YL, Liu L, Li MY, et al. Psychological disorders and psychosocial resources of patients with newly diagnosed bladder and kidney cancer: a cross-sectional study. PLoS One 2016;11:e0155607.

14. Zhu L, Ranchor AV, van der Lee M, et al. The role of goal adjustment in symptoms of depression, anxiety and fatigue in cancer patients receiving psychosocial care: a longitudinal study. Psychol Health 2015;30:268-83.

15. White VM, Macvean ML, Grogan S, et al. Can a tailored telephone intervention delivered by volunteers reduce the supportive care needs, anxiety and depression of people with colorectal cancer? A randomised controlled trial. Psychooncology 2012;21:1053-62.

16. Friedenreich CM, Neilson HK, Farris MS, et al. Physical Activity and Cancer Outcomes: A Precision Medicine Approach. Clin Cancer Res 2016;22:4766-75.

17. Demark-Wahnefried W, Schmitz KH, Alfano CM, et al. Weight management and physical activity throughout the cancer care continuum. CA Cancer J Clin 2018;68:64-89.

18. Lahart IM, Metsios GS, Nevill AM, et al. Physical activity for women with breast cancer after adjuvant therapy. Cochrane Database Syst Rev 2018;1:CD011292.

19. Leitzmann M, Powers H, Anderson AS, et al. European Code against Cancer 4th Edition: Physical activity and cancer. Cancer Epidemiol 2015;39 Suppl 1:S46-55.

20. Broocks A, Pirke KM, Schweiger U, et al. Cyclic ovarian function in recreational athletes. J Appl Physiol (1985) 1990;68:2083-6.

21. Holmberg L, Norden T, Lindgren A, et al. Pre-operative oestradiol levels - relation to survival in breast cancer. Eur
J Surg Oncol 2001;27:152-6.

22. Holmes MD, Chen WY, Feskanich D, et al. Physical activity and survival after breast cancer diagnosis. JAMA 2005;293:2479-86.

23. Hamilton R, Miedema B, Macintyre L, et al. Using a positive self-talk intervention to enhance coping skills in breast cancer survivors: lessons from a community-based group delivery model. Curr Oncol 2011;18:e46-53.

24. El-Shami K, Oeffinger KC, Erb NL, et al. American Cancer Society Colorectal Cancer Survivorship Care Guidelines. CA Cancer J Clin 2015;65:428-55.

25. Oberoi DV, White V, Jefford M, et al. Caregivers' information needs and their 'experiences of care' during treatment are associated with elevated anxiety and depression: a cross-sectional study of the caregivers of renal cancer survivors. Support Care Cancer 2016;24:4177-86.

26. Liu J, Zong G, Zhang C, et al. Anxiety and serum catecholamines as predictors of survival and recurrence in hepatocellular carcinoma. Psychooncology 2017;26:1347-53.

27. Vodermaier A, Lucas S, Linden W, et al. Anxiety after diagnosis predicts lung cancer-specific and overall survival in patients with stage iii non-small cell lung cancer: a populationbased cohort study. J Pain Symptom Manage 2017;53:1057-65.

28. Laconi E, Tomasi C, Curreli F, et al. Early exposure to restraint stress enhances chemical carcinogenesis in rat liver. Cancer Lett 2000;161:215-20.

29. Young WS 3rd, Lightman SL. Chronic stress elevates enkephalin expression in the rat paraventricular and supraoptic nuclei. Brain Res Mol Brain Res 1992;13:111-7.

30. Clevenger CV, Furth PA, Hankinson SE, et al. The role of prolactin in mammary carcinoma. Endocr Rev 2003;24:1-27.

31. Sephton S, Spiegel D. Circadian disruption in cancer: a neuroendocrine-immune pathway from stress to disease? Brain Behav Immun 2003;17:321-8.

32. Sephton SE, Sapolsky RM, Kraemer HC, et al. Diurnal cortisol rhythm as a predictor of breast cancer survival. J Natl Cancer Inst 2000;92:994-1000.

33. Antoni MH, Lutgendorf SK, Cole SW, et al. The influence of bio-behavioural factors on tumour biology: pathways and mechanisms. Nat Rev Cancer 2006;6:240-8.

Cite this article as: Song B, Zhang Y, Wang Y, An X. Intensive patients' care program ameliorates anxiety and depression, and sustained anxiety correlates with worse overall survival in renal cell carcinoma patients underwent radical nephrectomy. Trans Cancer Res 2018;7(6):1601-1613. doi: 10.21037/tcr.2018.12.07 
Supplementary

Table S1 Specific interventions of physical training

\begin{tabular}{|c|c|c|}
\hline Physical training & Duration and frequency & Specific interventions \\
\hline Low intensity & 6 months & \\
\hline Relaxation & $30 \mathrm{~min}, 5$ times a week & $\begin{array}{l}\text { The participants lay on mats with pillows and blankets and were instructed in tensing and } \\
\text { relaxing major muscle groups, working from head to foot, stretching and respiration }\end{array}$ \\
\hline Massage & $30 \mathrm{~min}$, twice a week & Massage included scar tissue massage, acupuncture, cupping therapy and so on \\
\hline Strolling & 30 min, 5 times a week & Walking slowly in a park or neighborhood with the company of the family \\
\hline Moderate-intensity & 6 months & \\
\hline Warm-up & $20 \mathrm{~min}, 5$ times a week & $\begin{array}{l}\text { The warm-up consisted of dynamic exercises with the large muscle groups, leg press, back } \\
\text { extension, leg extension and leg curl }\end{array}$ \\
\hline Aerobics & $40 \mathrm{~min}, 5$ times a week & The aerobic exercises included jogging, swimming, cycling and shadowboxing \\
\hline $\begin{array}{l}\text { Cardiopulmonary } \\
\text { training }\end{array}$ & 30 min, 3 times a week & $\begin{array}{l}\text { Cardiopulmonary training involved rhythmic sports including rope skipping, tennis, table } \\
\text { tennis, badminton and square dances }\end{array}$ \\
\hline
\end{tabular}

Table S2 Comparison of baseline characteristics between patients with sustained anxiety and patients without sustained anxiety (assessed by HADS-A score)

\begin{tabular}{|c|c|c|c|}
\hline Items & Sustained anxiety patients $(\mathrm{N}=20)$ & Non-sustained anxiety patients $(\mathrm{N}=162)$ & $P$ value \\
\hline Age (years) & $59.3 \pm 9.9$ & $59.0 \pm 10.8$ & 0.931 \\
\hline Gender (male/female) & $12 / 8$ & $114 / 48$ & 0.343 \\
\hline Highest education & & & 0.090 \\
\hline Primary school or less & $5(25.0)$ & $77(47.5)$ & \\
\hline High school & $11(55.0)$ & $45(27.8)$ & \\
\hline Undergraduate & $3(15.0)$ & $32(19.8)$ & \\
\hline Graduate or above & $1(5.0)$ & $8(4.9)$ & \\
\hline Smoke & $7(35.0)$ & $13(65.0)$ & 0.392 \\
\hline Drink & $9(45.0)$ & $61(37.7)$ & 0.524 \\
\hline Hypertension & $8(40.0)$ & $52(32.1)$ & 0.478 \\
\hline Hyperlipidemia & $2(10.0)$ & $39(24.1)$ & 0.155 \\
\hline Diabetes & $0(0.0)$ & $13(8.0)$ & 0.189 \\
\hline Tumor side & & & 0.091 \\
\hline Lift & $6(30.0)$ & $81(50.0)$ & \\
\hline Right & $14(70.0)$ & $81(50.0)$ & \\
\hline Histological subtypes & & & 0.479 \\
\hline Clear cell & $18(90.0)$ & $136(84.0)$ & \\
\hline Other & $2(10.0)$ & $26(16.0)$ & \\
\hline TNM stage & & & 0.928 \\
\hline I & $6(30.0)$ & $47(29.0)$ & \\
\hline II & $11(55.0)$ & $85(52.5)$ & \\
\hline III & $3(15.0)$ & $30(18.5)$ & \\
\hline
\end{tabular}

Data were presented as mean value \pm standard deviation or count (percentage). Comparison was determined by $t$-test or Chi-square test. $P$ value $<0.05$ was considered significant. IPCP, intensive patient's care program; HADS-A score, Hospital Anxiety and Depression Scale anxiety score. 
Table S3 Comparison of baseline characteristics between s patients with sustained anxiety and patients without sustained anxiety (by SAS anxiety score)

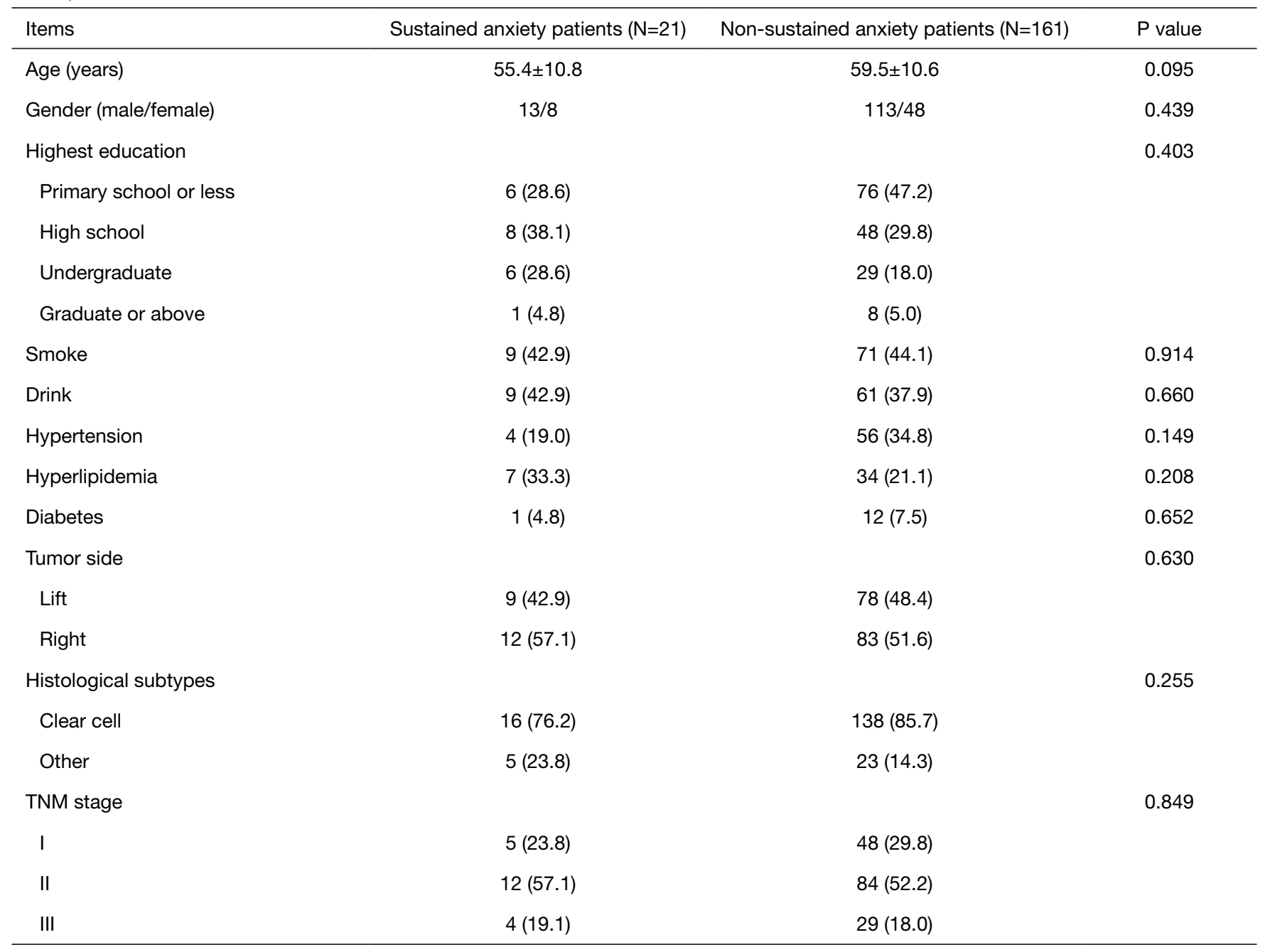

Data were presented as mean value \pm standard deviation or count (percentage). Comparison was determined by $t$ test or Chi-square test.

$P$ value $<0.05$ was considered significant. IPCP, intensive patient's care program; SAS, Zung Self-Rating Anxiety Scale. 